\title{
THE EFFECT OF FINITE ELEMENT DISCRETIZATION ON THE STATIONARY DISTRIBUTION OF SPDES*
}

\author{
JOCHEN VOSS ${ }^{\dagger}$
}

\begin{abstract}
This article studies the effect of discretization error on the stationary distribution of stochastic partial differential equations (SPDEs). We restrict the analysis to the effect of space discretization performed by finite element schemes. The main result is that under appropriate assumptions the stationary distribution of the finite element discretization converges in total variation norm to the stationary distribution of the full SPDE.
\end{abstract}

Key words. SPDEs, finite element discretization, stationary distribution.

AMS subject classifications. 60H35, 60H15, 65C 30 .

Introduction. In this article we consider the finite element discretization for stochastic partial differential equations (SPDEs) of the form

$$
\partial_{t} u(t, x)=\partial_{x}^{2} u(t, x)+f(u(t, x))+\sqrt{2} \partial_{t} w(t, x), \quad \forall(t, x) \in[0, \infty) \times[0,1],
$$

where $\partial_{t} w$ is space-time white noise and $f: \mathbb{R} \rightarrow \mathbb{R}$ is a smooth function with bounded derivatives, and where the differential operator $\partial_{x}^{2}$ is equipped with boundary conditions such that it is a negative operator on the space $L^{2}([0,1], \mathbb{R})$. More specifically, we are considering the effect that discretization of the SPDE has on its stationary distribution.

Our motivation for studying this problem lies in a recently proposed, SPDE-based sampling technique: when trying to sample from a distribution on path-space, e.g. in filtering/smoothing problems to sample from the conditional distribution of a process given some observations, one can do so using a Markov chain Monte Carlo approach. Such MCMC methods require a process with values in path-space, and it transpires that in some situations SPDEs of the form (0.1) can be used; see e.g. [11, 12] and [13] for a review. When implementing the resulting methods on a computer, the sampling SPDEs must be discretized and, because MCMC methods use the sampling process only as a source of samples from its stationary distribution, the effect of the discretization error on an MCMC method depends on how well the stationary distribution of the SPDE is approximated. While there are many results of approximation of trajectories of SPDEs [20,21, 14, 10, 16], approximation of the stationary distribution does not seem to be well-studied.

When discretizing an SPDE, discretization of space and time can be considered to be two independent problems. In cases where only the stationary distribution of the process is of interest, such as Metropolis sampling, using the next time step of the time discretization as a proposal will completely eliminate the error introduced by time discretization [2]. For this reason, in this article we restrict the analysis to the effect of space discretization alone. The discretization technique discussed here is a finite element discretization, which is a much-studied technique for deterministic PDEs. The approximation problem for stochastic PDEs, as studied in this article, differs from the deterministic case significantly, since here we have to compare the full

${ }^{*}$ Received: October 20, 2011; accepted (in revised form): February 10, 2012. Communicated by Tiejun Li.

${ }^{\dagger}$ School of Mathematics, University of Leeds, Leeds, LS2 9JT, UK (J.Voss@leeds.ac.uk). 
distribution of the solutions instead of considering the approximation of the solution as a function.

Finally, the error of the resulting sampling method is not only affected by the error in the stationary distribution, but also by the time it takes for the sampling equation to reach equilibrium. Here, we concentrate on the error in the equilibrium distribution itself and refer to [3] for discussion of the speed of convergence to equilibrium.

While the results of this article are formulated for SPDEs with values in $\mathbb{R}$, we expect the results and techniques to carry over to SPDEs with values in $\mathbb{R}^{d}, d>1$ without significant changes. We only restrict discussion to the one-dimensional case to ease notation. This is in contrast to the domain of the SPDEs: we consider the case of one spatial dimension because this is the relevant case for the sampling techniques discussed above, but this choice significantly affects the proofs and a different approach would likely be required to study the case of higher-dimensional spatial domains.

The text is structured as follows: In Section 1 we present the results required to characterize the stationary distribution of the SPDE (0.1). In Section 2 we introduce the finite element discretization scheme for (0.1) and identify the stationary distribution of the discretized equation. Building on these results, in Section 3, we state our main result about convergence of the discretized stationary distributions to the full stationary distribution. Finally, in Section 4, we give two examples in order to illustrate the link to the MCMC methods discussed above and also to demonstrate that the considered finite element discretization forms a concrete and easily implemented numerical scheme.

\section{The infinite-dimensional equation}

In order to study the SPDE (0.1), it is convenient to rewrite the equation as an evolution equation on the Hilbert space $\mathcal{H}=\mathrm{L}^{2}\left([0,1], \mathbb{R}^{d}\right)$; For a description of the underlying theory we refer, for example, to the monograph of [7]. We consider

$$
d u(t)=\mathcal{L} u(t) d t+f(u(t))+\sqrt{2} d w(t), \quad \forall t \geq 0,
$$

where the solution $u$ takes values in $\mathcal{H}$ and $f$ acts pointwise on $u$, i.e. $f(u)(x)=\tilde{f}(u(x))$ for almost all $x \in[0,1]$ for some function $\tilde{f}: \mathbb{R}^{d} \rightarrow \mathbb{R}^{d}$ such that $f$ maps $\mathcal{H}$ into itself. Furthermore, $w$ is an $\mathrm{L}^{2}$-cylindrical Wiener process and we equip the linear operator $\mathcal{L}=\partial_{x}^{2}$ with boundary conditions given by the domain

$$
\mathcal{D}(\mathcal{L})=\left\{u \in \mathrm{H}^{2}([0,1], \mathbb{R}) \mid \alpha_{0} u(0)-\beta_{0} \partial_{x} u(0)=0, \alpha_{1} u(1)+\beta_{1} \partial_{x} u(1)=0\right\}
$$

where $\alpha_{0}, \alpha_{1}, \beta_{0}, \beta_{1} \in \mathbb{R}$. The boundary conditions in (1.2) include the cases of Dirichlet $\left(\beta_{i}=0\right)$ and $\mathrm{v}$. Neumann $\left(\alpha_{i}=0\right)$ boundary conditions. The general case of $\alpha_{i}, \beta_{i} \neq 0$ is known as Robin boundary conditions.

We start our analysis by considering the linear equation

$$
d u(t)=\mathcal{L} u(t) d t+\sqrt{2} d w(t), \quad \forall t \geq 0 .
$$

For equation (1.3) to have a stationary distribution, we require $\mathcal{L}$ to be negative definite. The following lemma states necessary and sufficient conditions on $\alpha_{i}$ and $\beta_{i}$ for this to be the case. 
Lemma 1.1. The operator $\mathcal{L}$ is a self-adjoint operator on the Hilbert space $\mathcal{H}$. The operator $\mathcal{L}$ is negative definite if and only if $\alpha_{0}, \beta_{0}, \alpha_{1}, \beta_{1}$ are contained in the set

$$
\begin{aligned}
& A=\left\{\beta_{0}\left(\alpha_{0}+\beta_{0}\right)>0, \beta_{1}\left(\alpha_{1}+\beta_{1}\right)>0,\left|\left(\alpha_{0}+\beta_{0}\right)\left(\alpha_{1}+\beta_{1}\right)\right|>\left|\beta_{0} \beta_{1}\right|\right\} \\
& \cup\left\{\beta_{0}=0, \alpha_{0} \neq 0, \beta_{1}\left(\alpha_{1}+\beta_{1}\right)>0\right\} \cup\left\{\beta_{0}\left(\alpha_{0}+\beta_{0}\right)>0, \beta_{1}=0, \alpha_{1} \neq 0\right\} \\
& \cup\left\{\beta_{0}=0, \alpha_{0} \neq 0, \beta_{1}=0, \alpha_{1} \neq 0\right\} .
\end{aligned}
$$

Proof. From the definition of $\mathcal{L}$ it is easy to see that the operator is self-adjoint.

We have to show that $\mathcal{L}$ is negative if and only if $\left(\alpha_{0}, \beta_{0}, \alpha_{1}, \beta_{1}\right) \in A$. Without loss of generality we can assume $\beta_{i} \geq 0$ for $i=1,2$ and $\alpha_{i} \geq 0$ whenever $\beta_{i}=0$ (since we can replace $\left(\alpha_{i}, \beta_{i}\right)$ by $\left(-\alpha_{i},-\beta_{i}\right)$ if required). Assume that $\lambda$ is an eigenvalue of $\mathcal{L}$. If $\lambda>0$, the corresponding eigenfunctions are of the form

$$
u(x)=c_{1} \mathrm{e}^{\sqrt{\lambda} x}+c_{2} \mathrm{e}^{-\sqrt{\lambda} x},
$$

where $c_{1}$ and $c_{2}$ are given by the boundary conditions; for $u$ to be in the domain of $\mathcal{L}$, the coefficients $c_{1}$ and $c_{2}$ need to satisfy

$$
\left(\begin{array}{cc}
\alpha_{0}-\beta_{0} \sqrt{\lambda} & \alpha_{0}+\beta_{0} \sqrt{\lambda} \\
\left(\alpha_{1}+\beta_{1} \sqrt{\lambda}\right) \mathrm{e}^{\sqrt{\lambda}} & \left(\alpha_{1}-\beta_{1} \sqrt{\lambda}\right) \mathrm{e}^{-\sqrt{\lambda}}
\end{array}\right)\left(\begin{array}{l}
c_{1} \\
c_{2}
\end{array}\right)=\left(\begin{array}{l}
0 \\
0
\end{array}\right) .
$$

Non-trivial solutions exist only if the matrix is singular or, equivalently, if its determinant

$$
f(\lambda)=\alpha_{0} \alpha_{1}+\left(\alpha_{0} \beta_{1}+\alpha_{1} \beta_{0}\right) \sqrt{\lambda} \operatorname{coth} \sqrt{\lambda}+\beta_{0} \beta_{1} \lambda
$$

satisfies $f(\lambda)=0$. For $\lambda=0$ the eigenfunctions are of the form $u(x)=c_{1} 1+c_{2} x$, and an argument similar to the one above shows that the boundary conditions can be satisfied if and only if $\alpha_{0} \alpha_{1}+\alpha_{0} \beta_{1}+\alpha_{1} \beta_{0}=0$. Since $x \operatorname{coth}(x) \rightarrow 1$ as $x \rightarrow 0$, this condition can be written as $f(0)=0$, where

$$
f(0)=\lim _{\lambda \downarrow 0} f(\lambda)=\alpha_{0} \alpha_{1}+\alpha_{0} \beta_{1}+\alpha_{1} \beta_{0} .
$$

This shows that $\mathcal{L}$ is negative whenever $f(\lambda) \neq 0$ for all $\lambda \geq 0$.

Let $\left(\alpha_{0}, \beta_{0}, \alpha_{1}, \beta_{1}\right) \in A$. Assume first $\beta_{i} \neq 0$ for $i=1,2$ and let $\xi_{i}=\alpha_{i} / \beta_{i}$. Then, by the first condition in $A$, we have $\xi_{0}, \xi_{1}>-1$ and $\left(\xi_{0}-1\right)\left(\xi_{1}-1\right)>1$, and for $\lambda \geq 0$ we get

$$
f(\lambda)=\xi_{0} \xi_{1}+\left(\xi_{0}+\xi_{1}\right) \sqrt{\lambda} \operatorname{coth} \sqrt{\lambda}+\lambda \geq\left(\xi_{0}+1\right)\left(\xi_{1}+1\right)-1>0 .
$$

The cases $\beta_{0}=0$ or $\beta_{1}=0$ can be treated similarly. Thus, for $\left(\alpha_{0}, \beta_{0}, \alpha_{1}, \beta_{1}\right) \in A$ there are no eigenvalues with $\lambda \geq 0$ and the operator is negative.

For the converse statement, assume that $\left(\alpha_{0}, \beta_{0}, \alpha_{1}, \beta_{1}\right) \notin A$. We then have to show that there is a $\lambda \geq 0$ with $f(\lambda)=0$. Assume first $\beta_{i}>0$ for $i=1,2$ and define $\xi_{i}$ as above. If $\left(\xi_{0}+1\right)\left(\xi_{1}+1\right)=1$, we have $f(0)=0$. If $\left(\xi_{0}+1\right)\left(\xi_{1}+1\right)<1$, the function $f$ satisfies $f(0)<0$ and $f(\lambda) \rightarrow \infty$ as $\lambda \rightarrow \infty$; by continuity there is a $\lambda>0$ with $f(\lambda)=0$. Finally, if $\left(\xi_{0}+1\right)\left(\xi_{1}+1\right)>1$ but $\xi_{0}, \xi_{1} \leq-1$, we have $f(0)>0$ and for $\lambda$ with $\sqrt{\lambda}=-\left(\xi_{0}+\right.$ $\left.\xi_{1}\right) / 2>0$ we find $f(\lambda)<\xi_{0} \xi_{1}+\left(\xi_{0}+\xi_{1}\right) \sqrt{\lambda}+\lambda=\xi_{0} \xi_{1}-\left(\xi_{0}+\xi_{1}\right)^{2} / 4=-\left(\xi_{0}-\xi_{1}\right)^{2} / 4 \leq$ 
0 , so by continuity there is a $\lambda>0$ with $f(\lambda)=0$. Again, the cases $\beta_{0}=0$ and $\beta_{1}=0$ can be treated similarly.

A representation of the eigenvalues of $\mathcal{L}$ which is similar to the one in the proof of Lemma 1.1 can be found in Section 3 of [5].

The statement from Lemma 1.1 reproduces the well-known results that the Laplacian with Dirichlet boundary conditions $\left(\alpha_{i}=1, \beta_{i}=0\right)$ is negative definite whereas the Laplacian with von Neumann boundary conditions $\left(\alpha_{i}=0, \beta_{i}=1\right)$ is not (since constants are eigenfunctions with eigenvalue 0 ).

Lemma 1.2. Let $\mathcal{L}$ be negative definite. Then the following statements hold:

1. The linear SPDE (1.3) has global, continuous $\mathcal{H}$-valued solutions.

2. Equation (1.3) has a unique stationary distribution $\nu$ on $\mathcal{H}$. The measure $\nu$ is Gaussian with mean 0 and covariance function

$$
C(x, y)=\frac{\beta_{0} \beta_{1}+\alpha_{0} \beta_{1} x y+\beta_{0} \alpha_{1}(1-x)(1-y)}{\alpha_{0} \alpha_{1}+\alpha_{0} \beta_{1}+\beta_{0} \alpha_{1}}+x \wedge y-x y,
$$

where $x \wedge y$ denotes the minimum of $x$ and $y$.

3. The measure $\nu$ coincides with the distribution of $U \in \mathrm{C}([0,1], \mathbb{R})$ given by

$$
U(x)=(1-x) L+x R+B(x), \quad \forall x \in[0,1],
$$

where $L \sim \mathcal{N}\left(0, \sigma_{L}^{2}\right), R \sim \mathcal{N}\left(0, \sigma_{R}^{2}\right)$ with $\operatorname{Cov}(L, R)=\sigma_{L R}$, the process $B$ is a Brownian bridge, independent of $L$ and $R$, and

$$
\begin{aligned}
\sigma_{L}^{2} & =\frac{\beta_{0}\left(\alpha_{1}+\beta_{1}\right)}{\alpha_{0} \alpha_{1}+\alpha_{0} \beta_{1}+\beta_{0} \alpha_{1}}, \\
\sigma_{R}^{2} & =\frac{\left(\alpha_{0}+\beta_{0}\right) \beta_{1}}{\alpha_{0} \alpha_{1}+\alpha_{0} \beta_{1}+\beta_{0} \alpha_{1}}, \\
\sigma_{L R} & =\frac{\beta_{0} \beta_{1}}{\alpha_{0} \alpha_{1}+\alpha_{0} \beta_{1}+\beta_{0} \alpha_{1}} .
\end{aligned}
$$

Proof. From [15, 8] and [11] we know that (1.3) has global, continuous $\mathcal{H}$-valued solutions as well as a unique stationary distribution given by $\nu=\mathcal{N}\left(0,-\mathcal{L}^{-1}\right)$. An easy computation shows that $C$, as given in equation (1.5), is a Green's function for the operator $-\mathcal{L}$, i.e. $-\partial_{x}^{2} C(x, y)=\delta(x-y)$ and for every $y \in(0,1)$ the function $x \mapsto C(x, y)$ satisfies the boundary conditions (1.2). This completes the proof of the first two statements.

For the third statement we note that $U$ is centered Gaussian with covariance function

$$
\begin{aligned}
C(x, y) & =\operatorname{Cov}(U(x), U(y)) \\
& =\operatorname{Cov}((1-x) L+x R+B(x),(1-y) L+y R+B(y)) \\
& =(1-x)(1-y) \sigma_{L}^{2}+((1-x) y+x(1-y)) \sigma_{L R}+x y \sigma_{R}^{2}+x \wedge y-x y .
\end{aligned}
$$

The fact that this covariance function can be written in the form (1.5) can be checked by a direct calculation.

Using the results for the linear SPDE (1.3) we can now study the full SPDE (1.1). The result is given in the following lemma. 
Lemma 1.3. Let $\mathcal{L}$ be negative definite. Furthermore, let $f=F^{\prime}$, where $F \in \mathrm{C}^{2}(\mathbb{R}, \mathbb{R})$ is bounded from above with bounded second derivative. Then the following statements hold:

1. The nonlinear SPDE (1.1) has global, continuous $\mathcal{H}$-valued solutions.

2. Equation (1.1) has a unique stationary distribution $\mu$ which is given by

$$
\frac{d \mu}{d \nu}(u)=\frac{1}{Z} \exp \left(\int_{0}^{1} F(u(x)) d x\right)
$$

where $\nu$ is the stationary distribution of (1.3) from Lemma 1.2 and $Z$ is the normalisation constant.

Proof. This result is well known; see e.g. [22] or [12].

\section{Finite element approximation}

In this section we consider finite dimensional approximations of the SPDE (1.1), obtained by discretizing space using the finite element method. The approximation follows the same approach as for deterministic PDEs. For background on the deterministic case we refer to [4] or [17].

To discretize space, let $n \in \mathbb{N}, \Delta x=1 / n$, and consider $x$-values on the grid $k \Delta x$ for $k \in \mathbb{N}$. Since the differential operator $\mathcal{L}$ in (1.1) is a second order differential operator, we can choose a finite element basis consisting of "hat functions" $\varphi_{i}$ for $i \in \mathbb{Z}$ which have $\varphi_{i}(i \Delta x)=1, \varphi_{i}(j \Delta x)=0$ for all $j \neq i$, and which are affine between the grid points. Formally, the weak (in the PDE-sense) formulation of SPDE (1.1) can be written as

$$
\langle v, d u(t)\rangle=B(v, u) d t+\langle v, f(u(t))\rangle+\sqrt{2}\langle v, d w(t)\rangle,
$$

where $\langle\cdot, \cdot \cdot\rangle$ denotes the $\mathrm{L}^{2}$-inner product and the bilinear form $B$ is given by

$$
B(u, v)=\langle v, \mathcal{L} u\rangle=u(1) v^{\prime}(1)-u(0) v^{\prime}(0)-\int_{0}^{1} u^{\prime}(x) v^{\prime}(x) d x .
$$

The discretized solution is found by taking $u$ and $v$ to be in the space spanned by the functions $\varphi_{i}$, i.e. by using the ansatz

$$
u(t)=\sum_{j} U_{j}(t) \varphi_{j}
$$

and then considering the following system of equations:

$$
\left\langle\varphi_{i}, \sum_{j} d U_{j} \varphi_{j}\right\rangle=\left\langle\varphi_{i}, \partial_{x}^{2} \sum_{j} U_{j} \varphi_{j}\right\rangle d t+\left\langle\varphi_{i}, f\left(\sum_{j} U_{j} \varphi_{j}\right)\right\rangle d t+\sqrt{2}\left\langle\varphi_{i}, d w\right\rangle .
$$

The domain $V$ of the bilinear form $B$ depends on the boundary conditions of $\mathcal{L}$; there are four different cases:

1. If $\beta_{0}, \beta_{1} \neq 0$ in $(1.2)$, i.e. for von Neumann or Robin boundary conditions, we have $V=\mathrm{H}^{1}([0,1], \mathbb{R})$ and we consider the basis functions $\varphi_{i}$ for $i \in I=$ $\{0,1, \ldots, n-1, n\}$.

2. If $\beta_{0}=0$ and $\beta_{1} \neq 0$, i.e. for a Dirichlet boundary condition at the left boundary, we have $V=\left\{u \in \mathrm{H}^{1}([0,1], \mathbb{R}) \mid u(0)=0\right\}$ and we consider the basis functions $\varphi_{i}$ for $i \in I=\{1, \ldots, n-1, n\}$. 
3. If $\beta_{0} \neq 0$ and $\beta_{1}=0$, i.e. for a Dirichlet boundary condition at the right boundary, we have $V=\left\{u \in \mathrm{H}^{1}([0,1], \mathbb{R}) \mid u(1)=0\right\}$ and we consider the basis functions $\varphi_{i}$ for $i \in I=\{0,1, \ldots, n-1\}$.

4. If $\beta_{0}, \beta_{1}=0$, i.e. for Dirichlet boundary conditions at both boundaries, we have $V=\left\{u \in \mathrm{H}^{1}([0,1], \mathbb{R}) \mid u(0)=u(1)=0\right\}$ and we consider the basis functions $\varphi_{i}$ for $i \in I=\{1, \ldots, n-1\}$.

Throughout the rest of the text we will write $I$ for the index set of the finite element discretization as above, and the discretized solution $u=\sum_{j \in I} U_{j} \varphi_{j}$ will be described by the coefficient vector $U \in \mathbb{R}^{I}$. In all cases we define the "stiffness matrix" $L^{(n)} \in \mathbb{R}^{I \times I}$ by $L_{i j}^{(n)}=B\left(\varphi_{i}, \varphi_{j}\right)$ for all $i, j \in I$. For the given basis functions we get

$$
L_{i j}^{(n)}= \begin{cases}-\frac{2}{\Delta x} & \text { if } i=j \notin\{0, n\}, \\ +\frac{1}{\Delta x} & \text { if } i \in\{j-1, j+1\}, \\ -\frac{1}{\Delta x}-\frac{\alpha_{0}}{\beta_{0}} & \text { if } i=j=0, \\ -\frac{1}{\Delta x}-\frac{\alpha_{1}}{\beta_{1}} & \text { if } i=j=n, \\ 0 & \text { otherwise, }\end{cases}
$$

where the cases $i=j=0$ and $i=j=n$ cannot occur for Dirichlet boundary conditions. The "mass matrix" $M \in \mathbb{R}^{I \times I}$ is defined by $M_{i j}=\left\langle\varphi_{i}, \varphi_{j}\right\rangle$, and for $i, j \in I$ we get

$$
M_{i j}= \begin{cases}\frac{4}{6} \Delta x & \text { if } i=j \notin\{0, n\}, \\ \frac{1}{6} \Delta x & \text { if } i \in\{j-1, j+1\}, \\ \frac{2}{6} \Delta x & \text { if } i=j \in\{0, n\}, \\ 0 & \text { otherwise, }\end{cases}
$$

where again the cases $i=j=0$ and $i=j=n$ do not occur for Dirichlet boundary conditions. We note that the matrix $L^{(n)}$ only has the prefactor $1 / \Delta x$ instead of the $1 / \Delta x^{2}$ one would expect for a second derivative. The "missing" $\Delta x$ appears in the matrix $M$.

Since

$$
\operatorname{Cov}\left(\left\langle\varphi_{i}, w\right\rangle,\left\langle\varphi_{j}, w\right\rangle\right)=\left\langle\varphi_{i}, \varphi_{j}\right\rangle=M_{i j}
$$

Equation (2.1) can be written as

$$
M d U_{t}=L^{(n)} U_{t} d t+f_{n}\left(U_{t}\right) d t+\sqrt{2} M^{1 / 2} d W_{t},
$$

where $f_{n}: \mathbb{R}^{I} \rightarrow \mathbb{R}^{I}$ is defined by

$$
f_{n}(u)_{i}=\left\langle\varphi_{i}, f\left(\sum_{j \in I} u_{j} \varphi_{j}\right)\right\rangle
$$

for all $u \in \mathbb{R}^{I}$ and $i \in I$. Multiplication with $M^{-1}$ then yields the following SDE describing the evolution of the coefficients $\left(U_{i}\right)_{i \in I}$ :

DEFInITION 2.1. The finite element discretization of SPDE (1.1) is given by

$$
d U_{t}=M^{-1} L^{(n)} U_{t} d t+M^{-1} f_{n}\left(U_{t}\right) d t+\sqrt{2} M^{-1 / 2} d W_{t},
$$

where $W$ is an $|I|$-dimensional standard Brownian motion, $I \subseteq\{0,1, \ldots, n\}$ is the index set of the finite element discretization, and $L^{(n)}$ and $M$ are as above. 
Our aim is to show that the stationary distribution of (2.3) converges to the stationary distribution of the SPDE (1.1). We start our analysis by considering the linear case $f \equiv 0$. For this case the finite element discretization simplifies to (2.4) below.

Lemma 2.2. Let $\mathcal{L}$ be negative definite. Then $\nu_{n}=\mathcal{N}\left(0,\left(-L^{(n)}\right)^{-1}\right)$ is the unique stationary distribution of

$$
d U_{t}=M^{-1} L^{(n)} U_{t} d t+\sqrt{2} M^{-1 / 2} d W_{t} .
$$

Proof. Since $\mathcal{L}$ is a negative operator, the matrix $L^{(n)}$ is a symmetric, negative definite matrix. As the product of a positive definite symmetric matrix and a negative definite symmetric matrix, $M^{-1} L^{(n)}$ is negative definite; its eigenvalues coincide with the eigenvalues of

$$
M^{-1 / 2} L^{(n)} M^{-1 / 2}=-\left(\left(-L^{(n)}\right)^{1 / 2} M^{-1 / 2}\right)^{\top}\left(\left(-L^{(n)}\right)^{1 / 2} M^{-1 / 2}\right) .
$$

From [1, Theorem 8.2.12] we know that the unique stationary distribution of the $\operatorname{SDE}(2.4)$ is $\mathcal{N}\left(0, C^{(n)}\right)$, where $C^{(n)}$ solves the Lyapunov equation

$$
M^{-1} L^{(n)} C^{(n)}+C^{(n)} L^{(n)} M^{-1}=-2 M^{-1} .
$$

By Theorem 5.2.2 of [19], this system of linear equations has a unique solution, and it is easily verified that this solution is given by $C^{(n)}=\left(-L^{(n)}\right)^{-1}$.

The following lemma shows that for $f \equiv 0$ there is no discretization error at all: the stationary distributions of the $\operatorname{SPDE}(1.3)$, projected to $\mathbb{R}^{I}$, and of the finite element discretization (2.3) coincide.

Lemma 2.3. Define $\Pi: \mathrm{C}([0,1], \mathbb{R}) \rightarrow \mathbb{R}^{I}$ by

$$
(\Pi u)_{i}=u(i \Delta x), \quad \forall i \in I .
$$

Let $\nu$ be the stationary distribution of the linear SPDE $(1.3)$ on $\mathrm{C}([0,1], \mathbb{R})$, and let $\nu_{n}$ be the stationary distribution of the linear finite element discretization (2.4). Then we have

$$
\nu_{n}=\nu \circ \Pi^{-1}
$$

for every $n \in \mathbb{N}$.

Proof. Let $C^{\text {exact }}$ be the covariance matrix of $\nu \circ \Pi^{-1}$ and let $C^{(n)}$ be the covariance matrix of $\nu_{n}$. Since both measures under consideration are centered Gaussian, it suffices to show $C^{\text {exact }}=C^{(n)}$. By Lemma 1.2, the matrix $C^{\text {exact }}$ satisfies

$$
C_{i, j}^{\text {exact }}=C(i \Delta x, j \Delta x), \quad \forall i, j \in I,
$$

where $C$ is given by equation (1.5). By Lemma 2.2 we have $C^{(n)}=\left(-L^{(n)}\right)^{-1}$. A simple calculation, using the fact that both $C^{\text {exact }}$ and $L^{(n)}$ are known explicitly, shows $C^{\text {exact }} L^{(n)}=-I$ and thus $C^{\text {exact }}=C^{(n)}$ (the four different cases for the boundary conditions need to be checked separately). This completes the proof.

The preceding results only consider the linear case; for the general case, in the presence of the non-linearity $f$, we can of course no longer expect a similar result to 
hold. As a starting point for analyzing this case, we reproduce a well-known result which allows us to identify the stationary distribution of the discretized finite element equation.

Lemma 2.4. Let $F \in \mathrm{C}^{2}\left(\mathbb{R}^{d}, \mathbb{R}\right)$ have bounded second derivatives and satisfy the condition $Z=\int_{\mathbb{R}^{d}} \mathrm{e}^{2 F(x)} d x<\infty$. Furthermore, let $A \in \mathbb{R}^{d \times d}$ be invertible. Then the $S D E$

$$
d X_{t}=A A^{\top} \nabla F\left(X_{t}\right) d t+A d W_{t}
$$

has a unique stationary distribution which has density

$$
\varphi(x)=\frac{1}{Z} \mathrm{e}^{2 F(x)}
$$

with respect to the Lebesgue measure on $\mathbb{R}^{d}$.

Proof. Define $G(y)=F(A y)$ for all $y \in \mathbb{R}^{d}$. By the assumptions on $F$ we have $G \in$ $\mathrm{C}^{2}\left(\mathbb{R}^{d}, \mathbb{R}\right)$ with bounded second derivatives and $Z_{G}=\int_{\mathbb{R}^{d}} \mathrm{e}^{2 G(y)} d y<\infty$. Therefore, the $\mathrm{SDE}$

$$
d Y_{t}=\nabla G\left(Y_{t}\right) d t+d W_{t}
$$

has a unique stationary distribution with density

$$
\psi(y)=\frac{1}{Z_{G}} \mathrm{e}^{2 G(y)} .
$$

Since $\nabla G(y)=A^{\top} \nabla F(A y)$, we have

$$
d Y_{t}=A^{\top} \nabla F\left(A Y_{t}\right) d t+d W_{t}
$$

and multiplying this equation by $A$ gives

$$
d\left(A Y_{t}\right)=A A^{\top} \nabla F\left(A Y_{t}\right) d t+A d W_{t} .
$$

Consequently, $X_{t}=A Y_{t}$ satisfies the $\operatorname{SDE}(2.6)$ and has a unique stationary distribution with density proportional to $\psi\left(A^{-1} x\right) \propto \mathrm{e}^{2 G\left(A^{-1} x\right)}=\mathrm{e}^{2 F(x)}$. Since this function, up to a multiplicative constant, coincides with $\varphi$, the process $X$ has stationary density $\varphi$.

Because the stationary distribution in the lemma does not depend on $A$, the stationary distribution of (2.6) does not change when we remove/add $A$ from the equation. The process of introducing the matrix $A$ is sometimes called "preconditioning the SDE".

In cases where we are only interested in the stationary distribution of a discretized SPDE, the argument from Lemma 2.4 allows us to omit the mass matrix $M$ from the finite element SDE (2.3). In particular we do not need to consider the potentially computationally expensive square root $M^{1 / 2}$ in numerical simulations.

Lemma 2.5. Let $\mathcal{L}$ be negative definite. Furthermore, let $f=F^{\prime}$, where $F \in \mathrm{C}^{2}(\mathbb{R}, \mathbb{R})$ is bounded from above with bounded second derivative. Then the finite element SDE (2.3) has a unique stationary distribution $\mu_{n}$ given by

$$
\frac{d \mu_{n}}{d \nu_{n}}=\frac{1}{Z_{n}} \exp \left(F_{n}\right)
$$


where

$$
F_{n}(u)=\int_{0}^{1} F\left(\sum_{j \in I} u_{j} \varphi_{j}(x)\right) d x, \quad \forall u \in \mathbb{R}^{I},
$$

$Z_{n}$ is the normalisation constant and $\nu_{n}$ is the stationary distribution of the linear equation from Lemma 2.2.

Proof. Let $\Phi(u)=\frac{1}{2} u^{\top} L^{(n)} u+F_{n}(u)$ for all $u \in \mathbb{R}^{I}$. Then

$$
\partial_{i} \Phi(u)=\left(L^{(n)} u\right)_{i}+\left\langle\varphi_{i}, F^{\prime}\left(\sum_{j \in I} u_{j} \varphi_{j}\right)\right\rangle=\left(L^{(n)} u+f_{n}(u)\right)_{i}
$$

for all $i \in I$, and thus (2.3) can be written as

$$
d U_{t}=M^{-1} \nabla \Phi\left(U_{t}\right) d t+\sqrt{2} M^{-1 / 2} d W_{t} .
$$

By Lemma 2.4, this SDE has a unique stationary distribution $\mu_{n}$ whose density with respect to the $|I|$-dimensional Lebesgue measure $\lambda$ is given by

$$
\frac{d \mu_{n}}{d \lambda}(u)=\frac{1}{\tilde{Z}_{n}} \mathrm{e}^{\Phi(u)}=\frac{1}{\tilde{Z}_{n}} \exp \left(-\frac{1}{2} u^{\top}\left(-L^{(n)}\right) u+F_{n}(u)\right) .
$$

From Lemma 2.2 we know that the density of $\nu_{n}$ with respect to $\lambda$ is

$$
\frac{d \nu_{n}}{d \lambda}(x)=\frac{1}{(2 \pi)^{|I| / 2}\left(\operatorname{det}\left(-L^{(n)}\right)\right)^{\frac{1}{2}}} \exp \left(-\frac{1}{2} x^{\mathrm{T}}\left(-L^{(n)}\right) x\right),
$$

and consequently the distribution $\mu_{n}$ satisfies

$$
\frac{d \mu_{n}}{d \nu_{n}}(x)=\frac{d \mu_{n}}{d \lambda}(x) / \frac{d \nu_{n}}{d \lambda}(x) \propto \exp \left(F_{n}\right) .
$$

Since the right-hand side, up to constants, coincides with the expression in (2.7), the proof is complete.

\section{Main result}

Now that we have identified the stationary distribution of the SPDE (in Section 1) and of the SDE (in Section 2), we can compare the two stationary distributions. The result is given in the following theorem.

THEOREM 3.1. Let $\mu$ be the stationary distribution of the SPDE $(1.1)$ on $\mathrm{C}([0,1], \mathbb{R})$. Let $\mu_{n}$ be the stationary distribution of the finite element equation (2.3) on $\mathbb{R}^{I}$. Let $\mathcal{L}$ be negative and assume $f=F^{\prime}$, where $F \in \mathrm{C}^{2}(\mathbb{R})$ is bounded from above with bounded second derivative. Then

$$
\left\|\mu \circ \Pi^{-1}-\mu_{n}\right\|_{\mathrm{TV}}=\mathcal{O}\left(\frac{1}{n}\right)
$$

as $n \rightarrow \infty$, where $\|\cdot\|_{\mathrm{TV}}$ denotes total-variation distance between probability distributions on $\mathbb{R}^{I}$.

Before we prove this theorem, we first show some auxiliary results. The following lemma will be used to get rid of the (not explicitly known) normalization constant $Z_{n}$. 
Lemma 3.2. Let $(\Omega, \mathcal{F}, \mu)$ be a measure space and $f_{1}, f_{2}: \Omega \rightarrow[0, \infty]$ integrable with $Z_{i}=\int f_{i} d \mu>0$ for $i=1,2$. Then

$$
\int\left|\frac{f_{1}}{Z_{1}}-\frac{f_{2}}{Z_{2}}\right| d \mu \leq \frac{2}{\max \left(Z_{1}, Z_{2}\right)} \int\left|f_{1}-f_{2}\right| d \mu
$$

Proof. Using the $\mathrm{L}^{1}$-norm $\|f\|=\int|f| d \mu$ we can write

$$
\left\|\frac{f_{1}}{Z_{1}}-\frac{f_{2}}{Z_{2}}\right\| \leq\left\|\frac{f_{1}}{Z_{1}}-\frac{f_{2}}{Z_{1}}\right\|+\left\|\frac{f_{2}}{Z_{1}}-\frac{f_{2}}{Z_{2}}\right\|=\frac{1}{Z_{1}}\left\|f_{1}-f_{2}\right\|+\frac{\left|Z_{2}-Z_{1}\right|}{Z_{1} Z_{2}}\left\|f_{2}\right\| .
$$

Since $Z_{i}=\left\|f_{i}\right\|$ we can conclude

$$
\left\|\frac{f_{1}}{Z_{1}}-\frac{f_{2}}{Z_{2}}\right\| \leq \frac{1}{Z_{1}}\left\|f_{1}-f_{2}\right\|+\frac{\left|\left\|f_{2}\right\|-\left\|f_{1}\right\|\right|}{Z_{1}} \leq \frac{2}{Z_{1}}\left\|f_{1}-f_{2}\right\|,
$$

where the second inequality comes from the inverse triangle inequality. Without loss of generality we can assume $Z_{1} \geq Z_{2}$ (otherwise interchange $f_{1}$ and $f_{2}$ in the above argument), and thus the claim follows.

Lemma 3.3. Let $\mu$ and $\nu$ be probability measures on $\mathrm{C}([0,1], \mathbb{R})$ with $\mu \ll \nu$ and let $\Pi: \mathrm{C}([0,1], \mathbb{R}) \rightarrow \mathbb{R}^{I}$ be the projection from (2.5). Then $\mu \circ \Pi^{-1} \ll \nu \circ \Pi^{-1}$ and

$$
\frac{d\left(\mu \circ \Pi^{-1}\right)}{d\left(\nu \circ \Pi^{-1}\right)} \circ \Pi=\mathbb{E}_{\nu}\left(\frac{d \mu}{d \nu} \mid \Pi\right) .
$$

Proof. Let $\varphi=\frac{d \mu}{d \nu}$. Since $\mathbb{E}(\varphi \mid \Pi)$ is $\Pi$-measurable, there is a function $\psi: \mathbb{R} \rightarrow \mathbb{R}$ with $\mathbb{E}(\varphi \mid \Pi)=\psi \circ \Pi$. Let $A \subseteq \mathbb{R}^{I}$ be measurable. Then

$$
\begin{aligned}
\int_{\mathbb{R}} \psi 1_{A} d\left(\nu \circ \Pi^{-1}\right)=\int_{\mathrm{C}([0,1], \mathbb{R})} \psi \circ \Pi 1_{\Pi^{-1}(A)} d \nu \\
=\int_{\mathrm{C}([0,1], \mathbb{R})} \mathbb{E}(\varphi \mid \Pi) 1_{\Pi^{-1}(A)} d \nu=\int_{\mathrm{C}([0,1], \mathbb{R})} \varphi 1_{\Pi^{-1}(A)} d \nu=\mu \circ \Pi^{-1}(A)
\end{aligned}
$$

by the definition of conditional expectation. This shows that $\psi$ is indeed the required density.

Proof. (of Theorem 3.1.) Let $\Pi, \nu$, and $\nu_{n}$ be as in Lemma 2.3. Using Lemma 2.3 and 3.3 we find

$$
\begin{aligned}
\left\|\mu \circ \Pi^{-1}-\mu_{n}\right\|_{\mathrm{TV}} & =\mathbb{E}_{\nu_{n}}\left|\frac{d \mu \circ \Pi^{-1}}{d \nu_{n}}-\frac{d \mu_{n}}{d \nu_{n}}\right| \\
& =\mathbb{E}_{\nu}\left|\frac{d \mu \circ \Pi^{-1}}{d \nu \circ \Pi \Pi^{-1}} \circ \frac{d \mu_{n}}{d \nu_{n}} \circ \Pi\right| \\
& =\mathbb{E}_{\nu}\left|\mathbb{E}_{\nu}\left(\frac{d \mu}{d \nu}-\frac{d \mu_{n}}{d \nu_{n}} \circ \Pi \mid \Pi\right)\right| \\
& \leq \mathbb{E}_{\nu}\left|\frac{d \mu}{d \nu}-\frac{d \mu_{n}}{d \nu_{n}} \circ \Pi\right| .
\end{aligned}
$$

From Lemma 1.3 we know

$$
\frac{d \mu}{d \nu}(U)=\frac{1}{Z} \exp \left(\int_{0}^{1} F\left(U_{x}\right) d x\right) .
$$


Lemma 2.5 gives

$$
\frac{d \mu_{n}}{d \nu_{n}}=\frac{1}{Z_{n}} \exp \left(F_{n}\right)
$$

and by the definition of $F_{n}$ we have

$$
\frac{d \mu_{n}}{d \nu_{n}} \circ \Pi(U)=\frac{1}{Z_{n}} \exp \left(\int_{0}^{1} F\left(U_{x}^{(n)}\right) d x\right)
$$

where $U_{x}^{(n)}=\sum \Pi(U)_{j} \varphi_{j}(x)$ for all $U \in \mathrm{C}([0,1], \mathbb{R})$ and $\varphi_{j}, j \in I$ are the finite element basis functions. Using Lemma 3.2 we get

$$
\begin{aligned}
\left\|\mu \circ \Pi^{-1}-\mu_{n}\right\|_{\mathrm{TV}} & \leq \mathbb{E}_{\nu}\left|\frac{d \mu}{d \nu}-\frac{d \mu_{n}}{d \nu_{n}} \circ \Pi\right| \\
& \leq \frac{2}{Z} \int\left|\exp \left(\int_{0}^{1} F\left(U_{x}\right) d x\right)-\exp \left(\int_{0}^{1} F\left(U_{x}^{(n)}\right) d x\right)\right| d \nu(U) .
\end{aligned}
$$

Since the inequality $\left|\mathrm{e}^{x}-1\right| \leq|x| \exp (|x|)$ holds for all $x \in \mathbb{R}$, we conclude

$$
\begin{aligned}
&\left\|\mu \circ \Pi^{-1}-\mu_{n}\right\|_{\mathrm{TV}} \\
& \leq \frac{2}{Z} \mathbb{E}\left(\exp \left(\int_{0}^{1} F\left(U_{x}^{(n)}\right) d x\right) \cdot\left|\exp \left(\int_{0}^{1} F\left(U_{x}\right)-F\left(U_{x}^{(n)}\right) d x\right)-1\right|\right) \\
& \leq \frac{2}{Z} \mathbb{E}\left(\exp \left(\int_{0}^{1} F\left(U_{x}^{(n)}\right) d x\right)\right. \\
&\left.\cdot\left|\int_{0}^{1} F\left(U_{x}\right)-F\left(U_{x}^{(n)}\right) d x\right| \cdot \exp \left(\left|\int_{0}^{1} F\left(U_{x}\right)-F\left(U_{x}^{(n)}\right) d x\right|\right)\right)
\end{aligned}
$$

where $U$ is distributed according to the Gaussian measure $\nu$. Since $F$ is bounded from above we can estimate the the first exponential in the expectation by a constant. Using the Cauchy-Schwarz inequality we get

$$
\begin{aligned}
& \left\|\mu \circ \Pi^{-1}-\mu_{n}\right\|_{\mathrm{TV}} \\
\leq & c_{1}\left\|\int_{0}^{1} F\left(U_{x}\right)-F\left(U_{x}^{(n)}\right) d x\right\|_{2} \cdot\left\|\exp \left(\left|\int_{0}^{1} F\left(U_{x}\right)-F\left(U_{x}^{(n)}\right) d x\right|\right)\right\|_{2}
\end{aligned}
$$

for some constant $c_{1}$.

The main step in the proof is to estimate the right-hand side of 3.2 by showing that $\left|\int_{0}^{1} F\left(U_{x}\right)-F\left(U_{x}^{(n)}\right) d x\right|$ gets small as $n \rightarrow \infty$. By Lemma 1.2, the path $U$ in stationarity is just a Brownian bridge (with random boundary values) and, by definition, $U^{(n)}$ is the linear interpolation of the values of $U$ at the grid points (see figure 3.1 for illustration). Thus, the difference $U^{(n)}-U$ can be written as

$$
\left(U-U^{(n)}\right)(x)=\sum_{i=1}^{n} 1_{\left[\frac{i-1}{n}, \frac{i}{n}\right]}(x) \frac{1}{\sqrt{n}} B_{n x-(i-1)}^{(i)},
$$

where $B^{(1)}, \ldots, B^{(n)}$ are standard Brownian bridges, independent of each other and of $U^{(n)}$. Using the Taylor approximation for $F$ we find 


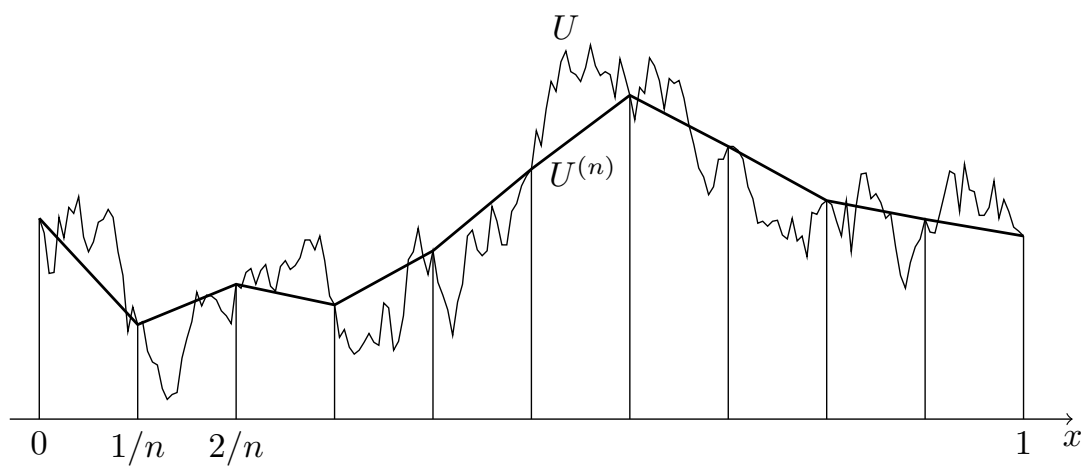

FIG. 3.1. Illustration of the convergence of $U^{(n)}$ to $U$. Under the distribution $\nu$, the path $U$ is a Brownian Bridge with random boundary conditions. Since $U^{(n)}$ is the linear interpolation of $U$ between the grid points, the difference $U^{(n)}-U$ consists of a chain of $n$ independent Brownian bridges.

$$
\begin{aligned}
& \left|\int_{0}^{1} F\left(U_{x}\right)-F\left(U_{x}^{(n)}\right) d x\right| \\
\leq & \left|\int_{0}^{1} f\left(U_{x}^{(n)}\right)\left(U_{x}-U_{x}^{(n)}\right) d x\right|+\frac{1}{2}\left\|F^{\prime \prime}\right\|_{\infty} \int_{0}^{1}\left(U_{x}-U_{x}^{(n)}\right)^{2} d x \\
= & :\left|P_{n}\right|+\frac{1}{2}\left\|F^{\prime \prime}\right\|_{\infty} Q_{n} .
\end{aligned}
$$

For the term $\left|P_{n}\right|$ we find

$$
P_{n}=\sum_{i=1}^{n} \int_{0}^{1 / n} f\left(U_{\frac{i-1}{n}+x}^{(n)}\right) \frac{1}{\sqrt{n}} B_{n x}^{(i)} d x=n^{-3 / 2} \sum_{i=1}^{n} \int_{0}^{1} f\left(U_{\frac{i-1}{n}+y / n}^{(n)}\right) B_{y}^{(i)} d y
$$

where $B^{(i)}$ are the Brownian bridges defined above. As an abbreviation write $\bar{f}_{i}(y)=$ $f\left(U_{\frac{i-1}{n}+y / n}^{(n)}\right)$. Conditioned on the value of $U^{(n)}$, the integrals $\int_{0}^{1} \bar{f}_{i}(y) B_{y}^{(i)} d y$ are centered Gaussian with

$$
\begin{aligned}
\operatorname{Var}\left(\int_{0}^{1} \bar{f}_{i}(y) B_{y}^{(i)} d y \mid U^{(n)}\right) & =\int_{0}^{1} \bar{f}_{i}(y) \int_{0}^{1}(y \wedge z-y z) \bar{f}_{i}(z) d z d y \\
& \leq c_{2}\left\|\bar{f}_{i}\right\|_{\infty}^{2} \leq c_{3}^{2}\left(\left\|U^{(n)}\right\|_{\infty}+1\right)^{2}
\end{aligned}
$$

for some constants $c_{2}, c_{3}>0$, where the last inequality uses the fact the $f$ is Lipschitz continuous. We get $\mathbb{E}\left(P_{n} \mid \Pi(U)\right)=0$ and, since the $B^{(i)}$ are independent,

$$
\mathbb{E}\left(P_{n}^{2} \mid U^{(n)}\right) \leq c_{3}^{2}\left(\left\|U^{(n)}\right\|_{\infty}+1\right)^{2} n^{-2} .
$$

Using the tower property for conditional expectations, and using $\left\|U^{(n)}\right\|_{\infty} \leq\|U\|_{\infty}$, we get

$$
\mathbb{E}\left(P_{n}^{2}\right) \leq c_{3}^{2} \mathbb{E}\left(\left(\|U\|_{\infty}+1\right)^{2}\right) n^{-2}
$$

Since $U$ is a Gaussian process, \|\|$U\left\|_{\infty}+1\right\|_{2}$ is finite, and we can conclude

$$
\mathbb{E}\left(P_{n}^{2}\right) \leq c_{4}^{2} n^{-2}
$$


for some constant $c_{4}$. Similarly, for $Q_{n}$ we find

$$
Q_{n}=\sum_{i=1}^{n} \int_{0}^{1}\left(B_{y}^{(i)}\right)^{2} d y \cdot n^{-2}
$$

and thus, using independence of the $B^{(i)}$ again,

$$
\mathbb{E}\left(Q_{n}^{2}\right)=c_{5}^{2} n^{-3}
$$

for some constant $c_{5}$. Combining these estimates we get

$$
\left\|\int_{0}^{1} F\left(U_{x}\right)-F\left(U_{x}^{(n)}\right) d x\right\|_{2}=\left\|P_{n}\right\|_{2}+\frac{1}{2}\left\|F^{\prime \prime}\right\|_{\infty}\left\|Q_{n}\right\|_{2} \leq c_{4} n^{-1}+c_{5} n^{-3 / 2},
$$

and thus we have shown the required bound for the first factor of (3.2).

Finally, we have to show that the second factor in (3.2) is bounded, uniformly in $n$ : From (3.3) we get

$$
\begin{aligned}
\left\|\exp \left(\left|\int_{0}^{1} F\left(U_{x}\right)-F\left(U_{x}^{(n)}\right) d x\right|\right)\right\|_{2} & \leq \mathbb{E}\left(\exp \left(2\left|P_{n}\right|+\left\|F^{\prime \prime}\right\|_{\infty} Q_{n}\right)\right)^{1 / 2} \\
& \leq\left\|\mathrm{e}^{2\left|P_{n}\right|}\right\|_{2}^{1 / 2} \cdot\left\|\mathrm{e}^{\left\|F^{\prime \prime}\right\|_{\infty} Q_{n}}\right\|_{2}^{1 / 2} .
\end{aligned}
$$

It is easy to check that, for all $\sigma>0$, an $\mathcal{N}\left(0, \sigma^{2}\right)$-distributed random variable $X$ satisfies the inequality $\mathbb{E}\left(\mathrm{e}^{|X|}\right) \leq 2 \mathrm{e}^{\sigma^{2} / 2}$, and thus we have

$$
\left\|\mathrm{e}^{2\left|P_{n}\right|}\right\|_{2}^{1 / 2}=\mathbb{E}\left(\mathrm{e}^{4\left|P_{n}\right|}\right)^{1 / 4} \leq 2 \exp \left(8 c_{4}^{2} n^{-2}\right)<1
$$

for all sufficiently large $n$. Furthermore, using (3.4) and the fact that the $B^{(i)}$ are i.i.d., we find

$$
\left\|\mathrm{e}^{\left\|F^{\prime \prime}\right\|_{\infty} Q_{n}}\right\|_{2}^{1 / 2}=\mathbb{E}\left(\exp \left(2\left\|F^{\prime \prime}\right\|_{\infty}\left|B^{(1)}\right|_{\mathrm{L}^{2}}^{2} n^{-2}\right)\right)^{n / 4},
$$

where we write $|\cdot|_{L^{2}}$ for the $\mathrm{L}^{2}$-norm on the space $\mathrm{L}^{2}([0,1], \mathbb{R})$. By Fernique's theorem [9] there exists an $\varepsilon>0$ with $\mathbb{E}\left(\exp \left(\varepsilon\left|B^{(1)}\right|_{\mathrm{L}^{2}}^{2}\right)\right)<\infty$. For all $\lambda>0$ we have

$$
\mathbb{E}\left(\left.\mathrm{e}^{\lambda \mid B^{(1)}}\right|_{\mathrm{L}^{2}} ^{2}\right)=\int_{0}^{\infty} \mathbb{P}\left(\left.\mathrm{e}^{\lambda \mid B^{(1)}}\right|_{\mathrm{L}^{2}} ^{2} \geq a\right) d a \leq 1+\int_{0}^{\infty} \mathbb{P}\left(\left|B^{(1)}\right|_{\mathrm{L}^{2}}^{2} \geq b\right) \lambda \mathrm{e}^{\lambda b} d b
$$

and using Markov's inequality $\mathbb{P}\left(\left|B^{(1)}\right|_{\mathrm{L}^{2}}^{2} \geq b\right) \leq \mathbb{E}\left(\left.\mathrm{e}^{\varepsilon \mid B^{(1)}}\right|_{\mathrm{L}^{2}} ^{2}\right) \mathrm{e}^{-\varepsilon b}$ we get

$$
\mathbb{E}\left(\left.\mathrm{e}^{\lambda \mid B^{(1)}}\right|_{\mathrm{L}^{2}} ^{2}\right) \leq 1+\int_{0}^{\infty} \mathbb{E}\left(\left.\mathrm{e}^{\varepsilon \mid B^{(1)}}\right|_{\mathrm{L}^{2}} ^{2}\right) \mathrm{e}^{-\varepsilon b} \lambda \mathrm{e}^{\lambda b} d b=1+\frac{\lambda}{\varepsilon-\lambda} \mathbb{E}\left(\left.\mathrm{e}^{\varepsilon \mid B^{(1)}}\right|_{\mathrm{L}^{2}} ^{2}\right)
$$

for all $\lambda<\varepsilon$. Substituting this bound into (3.8) we have

$$
\left\|\mathrm{e}^{\left\|F^{\prime \prime}\right\|_{\infty} Q_{n}}\right\|_{2}^{1 / 2} \leq\left(1+\frac{c_{6}}{n^{2}}\right)^{n} \leq 2
$$

for some constant $c_{6}$ and all sufficiently large $n$. From (3.7) and (3.9) we see that the right-hand side of (3.6) is bounded uniformly in $n$, i.e.

$$
\left\|\exp \left(\left|\int_{0}^{1} F\left(U_{x}\right)-F\left(U_{x}^{(n)}\right) d x\right|\right)\right\|_{2} \leq c_{7}
$$


for all $n \in \mathbb{N}$ and some constant $c_{7}$.

Combining (3.5) and (3.10) we see that the right-hand side in (3.2) is of or$\operatorname{der} \mathcal{O}\left(n^{-1}\right)$. This completes the proof.

For deterministic problems, the interpolation error of finite element methods can often be reduced by considering basis functions composed of higher-order polynomials instead of the linear basis functions considered here. This is a consequence of Céa's lemma, which states that the rate of error of the finite element approximation is determined by how well the exact solution can be approximated by the basis functions (see e.g. Theorem 2.8.1 in [4]). In contrast, when approximating Brownian motion by splines, the approximation error is always of order $1 / \sqrt{n}$ where $n$ is the number of spline-nodes, independent of the polynomial order of the splines [18,6]. For this reason we would expect that, for the situation considered here, the order of the leading error term $P_{n}$ in (3.3) cannot be improved by considering higher order polynomial basis functions.

In Theorem 3.1 we compared the stationary distribution $\mu_{n}$ of the finite element $\mathrm{SDE}$ on $\mathbb{R}^{I}$ and the stationary distribution $\mu$ of the $\operatorname{SPDE}$ on $\mathrm{C}([0,1], \mathbb{R})$ by projecting $\mu$ onto the finite dimensional space $\mathbb{R}^{I}$. An alternative approach is to embed $\mathbb{R}^{I}$ into $\mathrm{C}([0,1], \mathbb{R})$ instead. A naïve implementation of this idea would be to extend vectors from $\mathbb{R}^{I}$ to continuous functions via linear interpolation. Unfortunately, the image of $\mu_{n}$ when projected to $\mathrm{C}([0,1], \mathbb{R})$ in this way would be mutually singular with $\mu$ and thus the total variation norm would not provide a useful measure for the distance between the two distributions. For this reason, we choose here a different approach, described in the following definition.

Definition 3.4. Given a probability measure $\mu_{n}$ on $\mathbb{R}^{I}$, we define a distribution $\hat{\mu}_{n}$ as follows: Consider a random variable $X$ which is distributed according to $\mu_{n}$. Given $X$, construct $Y \in \mathrm{C}([0,1], \mathbb{R})$ by setting $Y(k \Delta x)=X_{k}$ for $k=0,1, \ldots, n$ and filling the gaps between these points with $n$ Brownian bridges, independent of $X$ and of each other. Then we denote the distribution of $Y$ by $\hat{\mu}_{n}$.

LEMMA 3.5. Let $\mu_{n}$ and $\nu_{n}$ be probability measures on $\mathbb{R}^{I}$ with $\mu_{n} \ll \nu_{n}$. Then $\hat{\mu}_{n} \ll \hat{\nu}_{n}$ with

$$
\frac{d \hat{\mu}_{n}}{d \hat{\nu}_{n}}=\frac{d \mu_{n}}{d \nu_{n}} \circ \Pi
$$

on $\mathrm{C}([0,1], \mathbb{R})$.

Proof. Let $\psi=\frac{d \mu_{n}}{d \nu_{n}}$. Using substitution we get

$$
\mathbb{E}_{\hat{\mu}_{n}}(f \circ \Pi)=\int_{\mathbb{R}^{I}} f d \mu_{n}=\int_{\mathbb{R}^{I}} f \psi d \nu_{n}=\mathbb{E}_{\hat{\nu}_{n}}(f \circ \Pi \cdot \psi \circ \Pi)
$$

for all integrable $f: \mathbb{R}^{I} \rightarrow \mathbb{R}$. Since, conditioned on the value of $\Pi$, the distributions $\hat{\mu}_{n}$ and $\hat{\nu}_{n}$ are the same, we can use the tower property to get

$$
\begin{aligned}
\hat{\mu}(A) & =\mathbb{E}_{\hat{\mu}_{n}}\left(\mathbb{E}_{\hat{\mu}_{n}}\left(1_{A} \mid \Pi\right)\right)=\mathbb{E}_{\hat{\mu}_{n}}\left(\mathbb{E}_{\hat{\nu}_{n}}\left(1_{A} \mid \Pi\right)\right) \\
& =\mathbb{E}_{\hat{\nu}_{n}}\left(\mathbb{E}_{\hat{\nu}_{n}}\left(1_{A} \mid \Pi\right) \cdot \psi \circ \Pi\right)=\mathbb{E}_{\hat{\nu}_{n}}\left(1_{A} \psi \circ \Pi\right)
\end{aligned}
$$

for every measurable set $A$. This shows that $\psi \circ \Pi$ is the required density. 
COROLlary 3.6. Let $\mu$ be the stationary distribution of the SPDE (1.1) on $\mathrm{C}([0,1], \mathbb{R})$. Let $\mu_{n}$ be the stationary distribution of the finite element equation (2.3) on $\mathbb{R}^{I}$. Let $\mathcal{L}$ be negative and assume $f=F^{\prime}$, where $F \in \mathrm{C}^{2}(\mathbb{R})$ is bounded from above with bounded second derivative. Then

$$
\left\|\mu-\hat{\mu}_{n}\right\|_{\mathrm{TV}}=\mathcal{O}\left(\frac{1}{n}\right)
$$

as $n \rightarrow \infty$.

Proof. Let $\nu$ be the stationary distribution of the linear SPDE (1.3) on $\mathrm{C}([0,1], \mathbb{R})$ and let $\nu_{n}$ be the stationary distribution of the linear finite element equation (2.4) on $\mathbb{R}^{I}$. By construction of the process $U$ in the third statement of Lemma 1.2 and by the Markov property for Brownian bridges, the distribution of $U$ between the grid points, conditioned on the values at the grid points, coincides with the distribution of $n$ independent Brownian bridges. By Lemma 2.3 the distribution of $U$ on the grid points is given by $\nu_{n}$. Thus we have $\nu=\hat{\nu}_{n}$. Using this equality and Lemma 3.5 we find

$$
\left\|\mu-\hat{\mu}_{n}\right\|_{\mathrm{TV}}=\mathbb{E}_{\nu}\left|\frac{d \mu}{d \nu}-\frac{d \hat{\mu}_{n}}{d \nu}\right|=\mathbb{E}_{\nu}\left|\frac{d \mu}{d \nu}-\frac{d \mu_{n}}{d \nu_{n}} \circ \Pi\right| .
$$

Now we are in the situation of equation (3.1), and the proof of Theorem 3.1 applies without further changes.

\section{Examples}

To illustrate that the suggested finite element method is a concrete and implementable scheme, this section gives two examples for the finite element discretization of SPDEs, both in the context of infinite dimensional sampling problems.

For the first example, fix $c>0$ and consider the SPDE

$$
\partial_{t} u(t, x)=\partial_{x}^{2} u(t, x)-c^{2} u(t, x)+\sqrt{2} \partial_{t} w(t, x), \quad \forall(t, x) \in \mathbb{R}_{+} \times(0,1),
$$

with Robin boundary conditions

$$
\partial_{x} u(t, 0)=c u(t, 0), \quad \partial_{x} u(t, 1)=-c u(t, 1), \quad \forall t \in \mathbb{R}_{+},
$$

where $\partial_{t} w$ is space-time white noise. From [12] we know that the stationary distribution of this SPDE on $\mathrm{C}([0,1], \mathbb{R})$ coincides with the distribution of the process $X$ given by

$$
\begin{aligned}
d X_{\tau} & =-c X_{\tau} d \tau+d W_{\tau}, \quad \forall \tau \in[0,1], \\
X_{0} & \sim \mathcal{N}\left(0, \frac{1}{2 c}\right),
\end{aligned}
$$

where the time $\tau$ in the SDE plays the rôle of the space $x$ in the SPDE. In the framework of Section 1, the boundary conditions (4.1) correspond to the case $\alpha_{0}=$ $\alpha_{1}=c$ and $\beta_{0}=\beta_{1}=1$. Since $\beta_{i} \neq 0$, we need to include both boundary points in the finite element discretization and thus have $I=\{0,1, \ldots, n\}$ and $\mathbb{R}^{I} \cong \mathbb{R}^{n+1}$. The matrix $L^{(n)}$ is given by

$$
L^{(n)}=\frac{1}{\Delta x}\left(\begin{array}{ccccc}
-1-c \Delta x & 1 & & & \\
1 & -2 & 1 & & \\
& 1 & -2 & 1 & \\
& & 1 & -2 & 1 \\
& & & 1 & -1-c \Delta x
\end{array}\right) \in \mathbb{R}^{(n+1) \times(n+1)}
$$


where the middle rows are repeated along the diagonal to obtain tridiagonal $(n+1) \times$ $(n+1)$-matrices. Similarly, the mass matrix $M$ is given by

$$
M=\frac{\Delta x}{6}\left(\begin{array}{rrrrr}
2 & 1 & & \\
1 & 4 & 1 & \\
& 1 & 4 & 1 \\
& 1 & 4 & 1 \\
& & 2
\end{array}\right) \in \mathbb{R}^{(n+1) \times(n+1)},
$$

where the middle rows are repeated along the diagonal. Finally, it transpires that the discretized drift for this example is given by $f_{n}(u)=-c M u$. By Lemma 2.4 the $n+1$ dimensional SDEs

$$
d U_{t}=M^{-1} L^{(n)} U_{t} d t-c U_{t} d t+\sqrt{2} M^{-1 / 2} d W_{t}
$$

and

$$
d U_{t}=L^{(n)} U_{t} d t-c M U_{t} d t+\sqrt{2} d W_{t},
$$

where $W$ is an $(n+1)$-dimensional Brownian motion, both have the same stationary distribution, and this stationary distribution converges to the distribution of the process $X$ from (4.2) in the sense given in Theorem 3.1 and Corollary 3.6.

As a second example, consider the SPDE

$$
\partial_{t} u(t, x)=\partial_{x}^{2} u(t, x)-\left(g g^{\prime}+\frac{1}{2} g^{\prime \prime}\right)(u)+\sqrt{2} \partial_{t} w(t, x), \quad \forall(t, x) \in \mathbb{R}_{+} \times(0,1),
$$

with Dirichlet boundary conditions

$$
u(t, 0)=u(t, 1)=0, \quad \forall t \in \mathbb{R}_{+},
$$

where $g \in \mathrm{C}^{3}(\mathbb{R}, \mathbb{R})$ has bounded derivatives $g^{\prime}, g^{\prime \prime}$, and $g^{\prime \prime \prime}$. From [12] we know that the stationary distribution of this $\operatorname{SPDE}$ on $\mathrm{C}([0,1], \mathbb{R})$ coincides with the conditional distribution of the process $X$ given by

$$
\begin{aligned}
d X_{\tau} & =g\left(X_{\tau}\right) d \tau+d W_{\tau}, \quad \forall \tau \in[0,1], \\
X_{0} & =0,
\end{aligned}
$$

conditioned on $X_{1}=0$.

Since we have Dirichlet boundary conditions, the boundary points in the finite element discretization are not included: we have $I=\{1,2, \ldots, n-1\}$ and $\mathbb{R}^{I} \cong \mathbb{R}^{n-1}$. The matrices $L^{(n)}$ and $M$ are given by

$$
L^{(n)}=\frac{1}{\Delta x}\left(\begin{array}{ccc}
-2 & 1 & \\
1 & -2 & 1 \\
& 1 & -2
\end{array}\right) \in \mathbb{R}^{(n-1) \times(n-1)}
$$

and

$$
M=\frac{\Delta x}{6}\left(\begin{array}{rrr}
4 & 1 \\
1 & 4 & 1 \\
1 & 4
\end{array}\right) \in \mathbb{R}^{(n-1) \times(n-1)} .
$$

where the middle rows are repeated along the diagonal to obtain matrices of the required size. The discretized drift $f_{n}$ can be computed from (2.2); if an analytical 
solution is not available, numerical integration can be used. By the assumptions on $g$, the function $F=-\frac{1}{2}\left(g^{2}+g^{\prime}\right)$ satisfies the conditions of Theorem 3.1. Thus, the stationary distributions of the $(n-1)$-dimensional SDEs

$$
d U_{t}=M^{-1} L^{(n)} U_{t} d t+M^{-1} f_{n}\left(U_{t}\right) d t+\sqrt{2} M^{-1 / 2} d W_{t}
$$

and

$$
d U_{t}=L^{(n)} U_{t} d t+f_{n}\left(U_{t}\right) d t+\sqrt{2} d W_{t}
$$

coincide and converge to the conditional distribution of $X$ from (4.3), conditioned on $X_{1}=0$.

\section{REFERENCES}

[1] L. Arnold, Stochastic Differential Equations: Theory and Applications, John Wiley \& Sons, 1974.

[2] A. Beskos, G.O. Roberts, A.M. Stuart, and J. Voss, MCMC methods for diffusion bridges, Stochastics and Dynamics, 8(3), 319-350, 2008.

[3] N. Bou-Rabee and M. Hairer, Non-asymptotic mixing of the MALA algorithm, IMA J. Numer. Anal., to appear, 2012.

[4] S.C. Brenner and L. Ridgway Scott, The Mathematical Theory of Finite Element Methods, Springer, second edition, 2002.

[5] C. Cacciapuoti and D. Finco, Graph-like models for thin waveguides with Robin boundary conditions, Asymp. Anal., 70(3-4), 199-230, 2010.

[6] J. Creutzig, T. Müller-Gronbach, and K. Ritter, Free-knot spline approximation of stochastic processes, J. Complexity, 23(4-6), 867-889, 2007.

[7] G. Da Prato and J. Zabczyk, Stochastic Equations in Infinite Dimensions, in Encyclopedia of Mathematics and its Applications, Cambridge University Press, 44, 1992.

[8] G. Da Prato and J. Zabczyk, Ergodicity for Infinite-Dimensional Systems, in London Mathematical Society Lecture Note Series, Cambridge University Press, 229, 1996.

[9] X. Fernique, Intégrabilité des vecteurs gaussiens, Comptes rendus hebdomadaires des séances de l'Académie des sciences, série A, 270, 1698-1699, 1970.

[10] I. Gyöngy and A. Millet, Rate of convergence of space time approximations for stochastic evolution equations, Pot. Anal., 30(1), 29-64, 2009.

[11] M. Hairer, A.M. Stuart, J. Voss, and P. Wiberg, Analysis of SPDEs arising in path sampling, Part I: The Gaussian case, Commun. Math. Sci., 3(4), 587-603, 2005.

[12] M. Hairer, A.M. Stuart, and J. Voss, Analysis of SPDEs arising in path sampling, Part II: The nonlinear case, Ann. App. Prob., 17(5), 1657-1706, 2007.

[13] M. Hairer, A.M. Stuart, and J. Voss, Sampling conditioned diffusions, London Mathematical Society Lecture Note Series, 353, 159-186, 2009.

[14] E. Hausenblas, Finite element approximation of stochastic partial differential equations driven by Poisson random measures of jump type, SIAM J. Numer. Anal., 46(1), 437-471, 2008.

[15] I. Iscoe, M.B. Marcus, D. McDonald, M. Talagrand, and J. Zinn, Continuity of $l^{2}$-valued Ornstein-Uhlenbeck processes, Ann. Prob., 18(1), 68-84, 1990.

[16] A. Jentzen, Higher order pathwise numerical approximations of SPDEs with additive noise, SIAM J. Numer. Anal., 49(2), 642-667, 2011.

[17] C. Johnson, Numerical Solution of Partial Differential Equations by the Finite Element Method, Cambridge University Press, 1990.

[18] M. Kon and L. Plaskota, Information-based nonlinear approximation: An average case setting, J. Complexity, 21(2), 211-229, 2005.

[19] P. Lancaster and L. Rodman, Algebraic Riccati Equations, Clarendon Press, Oxford, 1995.

[20] A. Millet and P.-L. Morien, On implicit and explicit discretization schemes for parabolic SPDEs in any dimension, Stoc. Proc. App., 115(7), 1073-1106, 2005.

[21] J.B. Walsh, Finite element methods for parabolic stochastic PDE's, Pot. Anal., 23(1), 1-43, 2005.

[22] J. Zabczyk, Symmetric solutions of semilinear stochastic equations, Stochastic partial differential equations and applications II, Giuseppe Da Prato and L. Tubaro (eds.), Lecture Notes in Mathematics, Springer, 1390, 237-256, 1989. 